\title{
Is correction of kyphosis necessary by posterior approach for the treatment of multilevel cervical degenerative myelopathy? An observational study
}

\section{Wei Du}

Hebei Medical University Third Affiliated Hospital

\section{Shuai Wang}

Hebei Medical University Third Affiliated Hospital

Haixu Wang

Hebei Medical University Third Affiliated Hospital

Jingtao Zhang

Hebei Medical University Third Affiliated Hospital

\section{Feng Wang}

Hebei Medical University Third Affiliated Hospital

\section{Xu Zhang}

Hebei Medical University Third Affiliated Hospital

\section{Yong Shen ( $\nabla$ shenyongspine@163.com )}

Hebei Medical University Third Affiliated Hospital

\section{Research article}

Keywords: multilevel cervical degenerative myelopathy; posterior approach; neurological improvement; kyphosis; axial symptoms

Posted Date: March 24th, 2020

DOI: https://doi.org/10.21203/rs.3.rs-18522/v1

License: (a) (1) This work is licensed under a Creative Commons Attribution 4.0 International License. Read Full License 


\section{Abstract}

Background: The purpose of this study was to determine whether sagittal lordotic alignment, clinical outcomes and axial symptoms (AS) could be improved by kyphotic correction after posterior approach for multilevel cervical degenerative myelopathy (CDM).

Methods: We retrospectively reviewed 109 patients with multilevel CDM with kyphosis who had undergone laminoplasty (Group LP, 53 patients) and laminectomy with lateral mass screw fixation (Group LCS, 56 patients) between January 2014 and December 2018. Curvature index (Cl) was measured according to the pre- and postoperative radiographic parameters. The recovery rate was calculated based on the Japanese Orthopedic Association (JOA) score. AS severity was quantified by Neck Disability Index (NDI).

Results: Analysis of postoperative follow-up data showed significant differences in $\mathrm{Cl}(\mathrm{t}=8.64, \mathrm{P}<$ $0.001)$, correction of $\mathrm{Cl}(\mathrm{t}=8.97, \mathrm{P}<0.001)$ and NDI $(\mathrm{t}=3.37, \mathrm{P}<0.001)$ between Group LP and LCS, whereas no significant differences in JOA score $(t=1.21, P=0.23)$ and recovery rate $(t=1.52, P=0.13)$. There were significant differences in JOA score $(\mathrm{t}=98.29,96.41, \mathrm{P}<0.001), \mathrm{Cl}(\mathrm{t}=17.07,16.17, \mathrm{P}<$ $0.001)$ and $\mathrm{NDI}(\mathrm{t}=37.46,52.15, \mathrm{P}<0.001)$ between pre- and postoperative follow-up in Group LP and LCS. Correction of $\mathrm{Cl}$ showed negative correlation with axial symptom severity $(r=-0.51, P<0.001)$, and no association with recovery rate $(r=0.14, P=0.15)$.

Conclusions: Satisfied neurological improvement was obtained by LP and LCS for patients with multilevel CDM, while kyphotic correction in Group LCS caused significant improvement of AS than that in Group LP. However, in a short-term postoperative follow-up, we could not demonstrate that kyphotic correction is associated with a better recovery in clinical outcomes.

\section{Background}

Cervical degenerative myelopathy (CDM), which can be debilitating to patients, causing neck pain, limb pain and numbness, is one of the most common causes of spinal dysfunction worldwide [1]. In patients with degenerative disease, cervical kyphosis is commonly seen because of the loss of sagittal lordotic alignment. Cervical kyphosis, which is the result of progressive degeneration of the discs and facet joints, is an uncommon but potentially debilitating and challenging condition [2].The posterior surgical approach has been considered as an effective and safe method for the treatment of multilevel CDM to widen the spinal canal dimensions with or without removing the dorsal elements of the cervical spine.

Laminoplasty is a standard surgical procedure to allow the posterior shift of the spinal cord for compressive myelopathy and provides good surgical result for long-term period. However, sagittal malalignment is one of the important potential complications following laminoplasty that may be associated with neck pain and functional disability $[3,4]$. If posterior decompression combined with instrumented fusion, the above problems may be resolved, but instrumentation failure (0\%-6\%), pseudarthrosis ( $0 \%-2 \%)$, infection, C5 nerve root palsy $(0 \%-25 \%)$ and vertebral artery injury $(0 \%-2 \%)$ were 
reported following fusion surgery [5-8]. The surgical strategy for multilevel CDM accompanying kyphosis remains controversial for the spine surgeons, due to the above shortcomings of each surgical approach.

We also conducted a literature search and found that there was significant debate on this issue, particularly as to whether sagittal lordotic alignment, clinical outcomes and axial symptoms could be improved by the correction of kyphosis. The purpose of this retrospective study was to determine whether sagittal lordotic alignment, clinical outcomes and axial symptoms could be improved by the correction of kyphosis after posterior approach for the treatment of multilevel CDM.

\section{Methods}

\section{Patient Population}

We retrospectively reviewed medical records of 109 patients with multilevel CDM associated with kyphosis who had undergone the laminoplasty (Group LP) and laminectomy with lateral mass screw fixation (Group LCS) at our medical center from January 2014 to December 2018. Patients in Group LP consisted of 53 cases, with a mean age of 62.5 years (range 51-76 years) who underwent expansive open-door laminoplasty. Patients in Group LC + Screw (LCS) included 56 patients, with a mean age of 63.5 years (range $52-74$ years) who underwent laminectomy with lateral mass screw fixation. The patients' age at the time of surgery ranged from 51 to 76 years (average 63.03 years), and the follow-up periods ranged from 10 to 18 months (average 13.37 months). This study was approved by the Investigational Review Board at our institution, and informed consent was obtained from each patient. All data regarding age, gender, decompressed levels, preoperative symptoms and follow-up period were reviewed and statistically analyzed (Table 1 ). 
Table 1

Patient Characteristics*

\begin{tabular}{|c|c|c|}
\hline Characteristics & Group LP & Group LCS \\
\hline Total $(n=109)$ & 53 & 56 \\
\hline Mean Age (years) & $51-76(62.5)$ & $52-74(63.5)$ \\
\hline \multicolumn{3}{|l|}{ Gender } \\
\hline Male & 37 & 39 \\
\hline Female & 16 & 17 \\
\hline \multicolumn{3}{|l|}{ Operation levels } \\
\hline 3 levels & 9 & 11 \\
\hline 4 levels & 15 & 18 \\
\hline 5 levels & 29 & 27 \\
\hline \multicolumn{3}{|l|}{ Presenting symptoms } \\
\hline \multicolumn{3}{|l|}{ Weakness } \\
\hline Upper extremity & 36 & 40 \\
\hline Lower extremity & 19 & 23 \\
\hline Extremity numbness hyperesthesia & 28 & 31 \\
\hline Gait instability & 35 & 33 \\
\hline Hyperreflexia & 39 & 41 \\
\hline Hoffman sign & 23 & 27 \\
\hline Babinski sign & 21 & 26 \\
\hline Clonus & 16 & 19 \\
\hline Follow-up time $(\mathrm{m})$ & $13.2(10-18)$ & $13.5(11-18)$ \\
\hline
\end{tabular}

Patients considered for the study had at least C3 levels of cervical spinal cord compression with accompanying symptoms and signs of cervical disc herniation, cervical spondylotic myelopathy, cervical spinal canal stenosis or segmental-type ossification of the posterior longitudinal ligament (OPLL). In addition, an alignment of $\mathrm{C} 2-7 \mathrm{Cobb}$ angle less than $0^{\circ}$ taken in lateral neutral radiograph was defined as kyphosis. Exclusion criteria included cases with cervical trauma or continuous-type OPLL, cases with significant cervical anatomic deformity, active infection, rheumatoid arthritis and neoplasm, cases whose 
pre- or postoperative follow-up magnetic resonance imaging (MRI) and plain radiographs were not complete or interpretable because of motion/metal artifacts or poor quality.

\section{Surgical technique}

All surgeries were performed by the same surgeon under general anesthesia. The open-door type of cervical en bloc laminoplasty described by Itoh and Tsuji [9] was performed in Group LP. One side of the lamina was opened, and the other side served as the hinge. Bone grafts from dissected spinous processes were put in the opened laminae and fixed with braided wires or nylon threads. In Group LCS, screws (Medtronic Sofamor Danek, Memphis, TN, USA) were placed bilaterally with the Magerl technique [10], rods of appropriate size were selected and bent to match the normal cervical lordosis and secured to the lateral masses by screws, and then laminectomy were performed based on the preoperative surgical planning. The patients were required to stay in bed for the first week after surgery, and thereafter walking was allowed with a Philadelphia neck collar for 6 weeks.

Before surgery, anteroposterior, lateral, and flexion/extension lateral X-ray tests and magnetic resonance imaging (MRI) scans were performed in patients. Routine X-ray tests were performed postoperatively at 3 , 6, 12 months and then 1.5 year. Patients underwent X-ray test in neutral standing position and were instructed to look straight ahead, with hips and knees extended.

\section{Radiological Assessments}

Pre- and postoperative follow-up cervical alignments were measured in the profile of neutral plain radiographs by curvature index $(\mathrm{Cl})$ as described by Ishihara [11] (Fig. 1). "a1" was defined as the distance from the posterior inferior edge of the $C 3$ vertebral body to line "AB", "a2, a3, and a4" using the same method. "AB" was defined as the distance from the posterior inferior edge of the $\mathrm{C} 2$ vertebral body to that of the $\mathrm{C} 7$ vertebral body.

Data measurements were performed three times with $200 \%$ magnification for accuracy by the first and second authors independently, and the mean value was used for analysis. The intraobserver errors were less than $5 \%$.

\section{Clinical Assessments}

The neurological status of each patient was evaluated at the pre- and postoperative follow-up according to the Japanese Orthopedic Association (JOA) disability scale. The neurological recovery rate was calculated using the Hirabayashi method [12]: (postoperative JOA score - preoperative score) / (17 preoperative score) $\times 100 \%$. Recovery rates were graded as follows: $\geq 75 \%$, excellent; $50-74 \%$, good; $25-$ $49 \%$, fair; and $<25 \%$, poor.

The severity of pre-and postoperative follow-up axial symptoms in each group was quantified by Neck Disability Index (NDI, $0=$ no disability, $50=$ total disability) [13]. Subjects' scores were calculated and ranked according to the standard NDI ranking system: $0-4$, no disability; $5-14$, mild disability; $15-24$, moderate disability; $25-34$, severe disability; $\geq 35$, complete disability [14]. 


\section{Statistical methods}

All statistical analysis was performed using Statistical Analysis System software (version 9.13, SAS Institute Inc., USA). Continuous variables were expressed as the means \pm SD. The Chi square test were applied for qualitative data. A paired $t$ test was used to assess statistical significance of changes between pre and postoperative parameters in each group. Statistical comparisons between Group LP and LCS were performed in the postoperative follow-up $\mathrm{Cl}$, JOA score, NDI score, correction of $\mathrm{Cl}$ and recovery rate using the independent sample t test, and in the severity of axial symptoms using Mann-Whitney $U$ test. Pearson's correlation coefficient was used to check the correlation among correction of $\mathrm{Cl}$, recovery rate and axial pain severity. A value of $P<0.05$ was considered to be statistically significant.

\section{Results}

\section{Radiographic results}

There was statistically significant difference between pre- and postoperative follow-up $\mathrm{Cl}(\mathrm{t}=17.07,16.17$, $P<0.001$ ) in Group LP and LCS (Table 2). There was also statistically significant difference at the postoperative follow-up $\mathrm{Cl}(\mathrm{t}=8.64, \mathrm{P}<0.001)$ and correction of $\mathrm{Cl}(\mathrm{t}=8.97, \mathrm{P}<0.001)$ between Group LP and LCS (Fig. 2).

Table 2

Pre- and postoperative follow-up cervical curvature index in each group

\begin{tabular}{|lllll|}
\hline Parameter & Group LP $(\mathbf{n}=\mathbf{5 3})$ & Group LCS $(\mathbf{n = 5 6 )}$ & t value * & P value \\
\hline Preoperative Cl (\%) & $8.73 \pm 2.20$ & $8.50 \pm 2.25$ & 0.53 & 0.60 \\
\hline Postoperative follow-up Cl (\%) & $11.38 \pm 2.44$ & $15.21 \pm 2.18$ & 8.64 & $<0.001$ \\
\hline Correction of Cl (\%) & $2.65 \pm 1.13$ & $6.71 \pm 3.10$ & 8.97 & $<0.001$ \\
\hline t value * & 17.07 & 16.17 & & \\
\hline P value & $<0.001$ & $<0.001$ & & \\
\hline * t test & & & & \\
\hline
\end{tabular}

\section{Functional results}

In this study, the pre- and postoperative follow-up JOA scores were 8.57/14.72 in Group LP, 8.46/14.96 in Group LCS, respectively. There were statistically significant differences between pre- and postoperative follow-up JOA scores $(t=98.29,96.41, P<0.001)$ in Group LP and LCS, while no significant differences in pre- and postoperative JOA scores between the two groups $(t=0.59, P=0.56 ; t=1.21, P=0.23)$. 
The neurological recovery was excellent in $28(52.83 \%$, LP), 38 (67.86\%, LCS) patients, good in 25 $(47.17 \%, \mathrm{LP}), 18$ (32.14\%, LCS), and there were no fair and poor cases in two groups. The improvement rate were $73.52 \% \pm 11.39 \%$ and $76.86 \% \pm 11.57 \%$ at the postoperative follow-up after laminoplasty and laminectomy with lateral mass screw fixation (Table 3 ). $t$ test showed no significant difference between the two groups for recovery rate $(t=1.52, P=0.13)$. Correction of curvature indexshowed no correlation with recovery rate $(r=0.14, P=0.15)(F i g .3)$.

Table 3

Pre- and postoperative follow-up JOA score and neurological recovery rate in each group

\begin{tabular}{|c|c|c|c|c|}
\hline Parameter & $\begin{array}{l}\text { Group LP ( }=53 \\
\text { ) }\end{array}$ & $\begin{array}{l}\text { Group LCS ( } n=56 \\
\text { ) }\end{array}$ & $\begin{array}{l}\text { Statistic } \\
\text { value }\end{array}$ & $\begin{array}{l}P \\
\text { value }\end{array}$ \\
\hline \multicolumn{5}{|l|}{ JOA score * } \\
\hline Preoperation & $8.57 \pm 0.84$ & $8.46 \pm 0.95$ & 0.59 & 0.56 \\
\hline Postoperative follow-up & $14.72 \pm 1.05$ & $14.96 \pm 1.10$ & 1.21 & 0.23 \\
\hline $\mathrm{t}$ value & 98.29 & 96.41 & & \\
\hline$P$ value & $<0.001$ & $<0.001$ & & \\
\hline \multicolumn{5}{|c|}{$\underset{\star \star}{\text { Neurological recovery rate grade }}$} \\
\hline Excellent ( $\geq 75 \%)$ & 28 & 38 & \multirow[t]{4}{*}{2.57} & \multirow[t]{4}{*}{0.11} \\
\hline Good (50-74\%) & 25 & 18 & & \\
\hline Fair (25-49\%) & 0 & 0 & & \\
\hline Poor $(<25 \%)$ & 0 & 0 & & \\
\hline Recovery rate $(\%)$ * & $73.52 \pm 11.39$ & $76.86 \pm 11.57$ & 1.52 & 0.13 \\
\hline \multicolumn{5}{|l|}{${ }^{*}$ t test } \\
\hline ** Pearson Chi-Square te & & & & \\
\hline
\end{tabular}

\section{Axial symptoms}

In the postoperative follow-up, NDI score was $6.77 \pm 6.21$ in Group LP and $3.52 \pm 3.60$ in Group LCS, respectively. According to the NDI ranking system, there was no disability in 29 patients, mild disability in 19 , moderate disability in 5, both severe disability and complete disability in 0 in the Group LP. Within Group LCS, the NDI ranking system indicated no disability in 45 patients, mild disability in 11 , and there were no moderate, severe and complete disability cases (Table 4). 
Axial symptom severity (NDI scores) in each group

\begin{tabular}{|c|c|c|c|c|}
\hline Axial symptoms & Group LP $(n=53)$ & Group LCS $(n=56)$ & Statistic value & $P$ value \\
\hline \multicolumn{5}{|l|}{ NDI score * } \\
\hline Preoperation & $20.45 \pm 4.60$ & $20.70 \pm 4.31$ & 0.29 & 0.78 \\
\hline Postoperative follow-up & $6.77 \pm 6.21$ & $3.52 \pm 3.60$ & 3.37 & $<0.001$ \\
\hline $\mathrm{t}$ value & 37.46 & 52.15 & & \\
\hline$P$ value & $<0.001$ & $<0.001$ & & \\
\hline \multicolumn{5}{|l|}{ NDI ranking system ** } \\
\hline No disability $(0-4)$ & 29 & 45 & 528.000 & $<0.001$ \\
\hline Mild disability (5-14) & 19 & 11 & & \\
\hline Moderate disability (15-24) & 5 & 0 & & \\
\hline Severe disability (25-34) & 0 & 0 & & \\
\hline Complete disability $(\geq 35)$ & 0 & 0 & & \\
\hline \multicolumn{5}{|l|}{${ }^{*}$ t test } \\
\hline ** Mann-Whitney U test & & & & \\
\hline
\end{tabular}

Axial symptom incidence was $45.28 \%$ (24/53 patients) in Group LP and 19.64\% (11/56 patients) in Group LCS, respectively. Mann-Whitney $U$ test showed significant difference between the two groups for axial symptoms $(U=528, P<0.001)$. Correction of curvature index showed negative correlation with axial symptom severity $(r=-0.51, P<0.001)$ (Fig. 4).

\section{Discussion}

Multilevel CDM, which is characterized by multisegmental spinal cord compression, is a common spinal disorder all over the world. Cervical kyphosis associated with multilevel CDM is the result of progressive subluxation of the apophyseal joints because of degenerative changes in the facet joints and discs [2]. Laminoplasty has been proved to be an effective and safe treatment for multisegmental lesions to widen the spinal canal without removing the dorsal elements of the cervical spine. In the past decades, lateral mass screws fixation has become optimal preferred option for stabilizing the cervical spine and correcting kyphotic deformity when multilevel decompression is required [15]. During the follow-up period, we also observed that some patients suffered the so-called axial symptoms including nuchal pain, neck stiffness and shoulder pain, which affect their quality of postoperative life seriously. The optimal surgical 
procedure for multilevel CDM accompanying kyphosis remains controversial due to the above shortcomings.

At the postoperative follow-up, correction of cervical kyphosis in Group LCS was better than that in Group LP; the difference was statistically significant between the groups. Based on the biomechanical study [16], the physiologic lordosis of the cervical spine plays an important role in maintaining normal neurological function. This emphasizes the importance of early recognition of complications caused by cervical curvature changes. There is controversy on the issue: Is the correction of cervical curvature related to neurological recovery rate and axial symptoms?

In the present study, better neurological improvement was both obtained in laminoplasty and laminectomy with fixation; there was no significant difference between groups LP and LCS. Our results in short-term follow-up did not demonstrated that correction of cervical kyphosis was correlated with neurological improvement. Zhang et al [2] suggested that loss of cervical lordosis after laminoplasty contributed to kyphotic alignment change, which prevented indirect decompression via posterior cervical spinal cord shift and led to postoperative residual anterior compression of cervical spinal cord; thus, progressive kyphosis results in late neurological deterioration in long-term follow-up of laminoplasty. Based on previous results [17] for an average 9.17 years' follow-up of 98 patients undergoing posterior operations, we revealed that loss of $\mathrm{Cl}$ was positively correlated with poor neurological recovery, especially in the patients with laminectomy alone, and presumed that segmental and kyphotic instability could be the main cause of poor neurological recovery in the long-term follow-up. Conversely, in the current study, we insisted that adequate decompression of the spinal cord in the operation may be a pivotal factor in early postoperative neurological recovery, which was not yet clearly associated with correction of cervical kyphosis. This result was in agreement with recent reports [18-20], indicating that there were no correlations between cervical sagittal alignment parameters and postoperative outcomes for the patients with maintained cervical lordosis who underwent posterior decompression and fusion at 1-year follow-up.

At the postoperative follow-up, 32.11\% (35/109) of the entire group experienced axial symptoms, an incidence consistent with previous studies. The incidence of axial symptoms was $45.28 \%(24 / 53)$ for group LP and 19.64\% (11/56) for group LCS, respectively; the difference was statistically significant. The incidence of axial symptoms after open-door laminoplasty can be as high as 5-86\% [21], but the exact etiological mechanism remains uncertain. Wang et al [22] believed that the destruction of cervical structures and detachment of posterior muscles, which increased cervical flexion mechanical stress, may play a pivotal role in the pathogenesis of AS. The spinous process, which was reconstructed firmly in the midline with titanium plates and enhanced it using nonabsorbable sutures with a high fusion rate and good stability, was beneficial for early postoperative exercises and improving cervical-spine activity; thereby, this may improve postoperative AS and decrease the loss of cervical range of motion [23]. Application of rigid internal fixation can reduce the incidence of advanced AS because it can enhance the stability of cervical vertebrae and reduce irritation in nearby soft tissues [24]. In present study, cervical kyphosis was corrected and reconstructed in Group LCS, whose incidence of axial symptoms was 
significantly lower than that in Group LP. The present study showed that axial symptom severity was negatively correlated with cervical kyphotic correction, which also meant that neck pain would be improved significantly if the cervical kyphosis was corrected effectively.

However, some patients at the postoperative follow-up did not complain of neck pain (group LP 29/53; group LCS 45/56). Although the patients of two groups complained more or less of neck pain after surgery in short time, neck pain was gradually improved with the correction of cervical lordosis and strengthening of cervical stability. Chen et al [25] insisted that LP with preservation of unilateral posterior muscle-ligament complex (PMLC) (including paravertebral muscles, as well as the nuchal ligament and attachment sites between extension muscles and spinous process) had the advantage of less soft-tissue detachment and provided greater stability, as well as better muscular alignment and reduced postoperative AS. There were ample studies $[26,27]$ demonstrating that reducing the invasion of the cervical extensor, retaining muscles attached to the spinous process significantly lowered the incidence of AS and decreased the loss of sagittal cervical lordosis after surgery. Moreover, early postoperative extensor muscle rehabilitation protocols all likely play a role in prevention or limitation of neck pain following laminoplasty [28]. Axial symptoms might also be caused by other problems, such as destruction of facet joints, intraoperative nerve root damage and hinge side nonunion. In short, axial symptoms were the results of a complication induced by multifactor and multimechanism after posterior cervical surgery, and the explicit pathogenesis remains to be further investigated.

Therefore, we thought that successful treatment of multilevel CDM with kyphosis required not only adequate decompression of the spinal cord, but also correction of cervical kyphosis as much as possible to avoid the surgery-related complications caused by sagittal malalignment or instability. There are several limitations need to be considered in our study. Over the last decade, many modified anterior or posterior surgical approaches for the treatment of multilevel CDM with kyphosis, including multilevel anterior cervical discectomy with fusion [29], modified unilateral open-door laminoplasty with PMLC preservation [25], laminoplasty with titanium miniplates fixation [30,31] and combined anterior- posterior fusion, had been developed and obtained favorable outcomes, and additionally reduced the incidence of the long-term surgery-related complications. This study still needs other parameters to comprehensively evaluate the results, including T1 slope, C2-7 ROM, C2-7 sagittal vertical axis (C2-7 SVA) and cephalad vertebral level undergoing laminoplasty (CVLL). Subsequently, there is still a need for prospective, largescale, multi-center clinical trials to further confirm our results.

\section{Conclusions}

Satisfied neurological improvement was obtained by LP and LCS for patients with multilevel CDM, while kyphotic correction in group LCS caused a significant improvement of axial symptoms. However, in a short-term postoperative follow-up, we could not demonstrate that the correction of kyphosis is associated with a better recovery in clinical outcomes.

\section{Declarations}




\section{Abbreviations}

MCDM: Multilevel cervical degenerative myelopathy; LP: Laminoplasty; LCS: Laminectomy with lateral mass screw fixation; Cl: Curvature index; JOA: Japanese Orthopedic Association; AS: Axial symptoms; NDI: Neck Disability Index; PMLC: Posterior muscle-ligament complex; SVA: sagittal vertical axis; OPLL: Ossification of the posterior longitudinal ligament.

\section{Ethics approval and consent to participate}

This study was approved by the Investigational Review Board at our institution, and informed consent was obtained from each patient.

\section{Consent for publication}

Not applicable.

\section{Availability of data and materials}

The datasets used and/or analyzed during the current study are available from the corresponding author on reasonable request.

\section{Competing interests}

All authors declare that they have no competing interests.

\section{Funding}

This study was funded by Natural Science Foundation of Hebei Province (No. H2017206371) and Health Commission of Hebei Province (No. 20180407).

\section{Authors' contributions}

WD was responsible for the conception and design of this study, as well as the acquisition and interpretation of data and preparation of a manuscript draft. SW, HW, JZ, and FW contributed to the imaging analysis and the acquisition and interpretation of data. XZ and YS critically revised the intellectual content of the manuscript and prepared the final version of the manuscript to be published. All of the authors have read and approved the final manuscript.

\section{Acknowledgments}

Not applicable.

\section{Author details}


${ }^{1}$ Department of Spine Surgery, The Third Hospital of Hebei Medical University, Shijiazhuang, 050051, People's Republic of China. ${ }^{2}$ Department of Hand Surgery, The Third Hospital of Hebei Medical University, Shijiazhuang, 050051, People's Republic of China.

\section{References}

1. Bakhsheshian J, Mehta VA, Liu JC. Current diagnosis and management of cervical spondylotic myelopathy. Global Spine J, 2017; 7(6): 572-586.

2. Zhang JT, Li JQ, Niu RJ, Liu Z, Tong T, Shen Y. Predictors of cervical lordosis loss after laminoplasty in patients with cervical spondylotic myelopathy. Eur Spine J, 2017; 26(4): 1205-1210.

3. Liu S, Yang SD, Fan XW, Yang DL, Ma L, Sun JY, Ding WY. Analyses of effect factors associated with the postoperative dissatisfaction of patients undergoing open-door laminoplasty for cervical OPLL: a retrospective cohort study. J Orthop Surg Res,2019; 14(1):161.

4. Ao $\mathrm{S}$, Liu $\mathrm{Y}$, Wang $\mathrm{Y}$, Zhang $\mathrm{H}$, Leng $\mathrm{H}$. Cervical kyphosis in asymptomatic populations: incidence, risk factors, and its relationship with health-related quality of life. J Orthop Surg Res,2019; 14(1): 322.

5. Kim BS, Dhillon RS. Cervical laminectomy with or without lateral mass instrumentation: a comparison of o Clin Spine Surg, 2019; 32(6): 226-232.

6. Coe JD, Vaccaro AR, Dailey AT, et al. Lateral mass screw fixation in the cervical spine: a systematic literature review. J Bone Joint Surg, 2013; 95(23): 2136-2143.

7. Basaran R, Kaner T. C5 nerve root palsy following decompression of cervical spine with anterior versus posterior types of procedures in patients with cervical myelopathy. Eur Spine J, 2016; 25(7): 2050-2059.

8. Molinari RW, Chimenti PC, Molinari R, Gruhn W. Vertebral artery injury during routine posterior cervical exposure: case reports and review of literature. Global Spine J, 2015; 5(6): 528-532.

9. Itoh T, Tsuji H. Technical improvements and results of laminoplasty for compressive myelopathy in the cervical spine. Spine (Phila Pa 1976), 1985; 10(8): 729-736.

10. Pal D, Bayley E, Magaji SA, Boszczyk BM. Freehand determination of the trajectory angle for cervical lateral mass screws: how accurate is it? Eur Spine J, 2011; 20(6): 972-976.

11. Takeshita K, Murakami M, Kobayashi A, Nakamura C. Relationship between cervical curvature index (Ishihara) and cervical spine angle (C2-7). J Orthop Sci, 2001; 6(3): 223-226.

12. Hirabayashi K, Miyakawa J, Satomi K, Maruyama T, Wakano K. Operative results and postoperative progression of ossification among patients with ossification of cervical posterior longitudinal ligament. Spine (Phila Pa 1976), 1981; 6(4): 354-364.

13. Vernon H, Mior S. The Neck Disability Index: a study of reliability and validity. J Manipulative Physiol Ther, 1991; 14(7): 409-415.

14. McCarthy MJ, Grevitt MP, Silcocks P, Hobbs G. The reliability of the Vernon and Mior neck disability index, and its validity compared with the short form-36 health survey questionnaire. Eur Spine J, 2007; 16(12): 2111-2117. 
15. Kurd MF, Millhouse PW, Schroeder GD, Kepler CK, Vaccaro AR. Lateral mass fixation in the subaxial cervical s J Spinal Disord Tech, 2015; 28(7): 259-263.

16. Patwardhan AG, Khayatzadeh S, Havey RM, et al. Cervical sagittal balance: a biomechanical perspective can help clinical practice. Eur Spine J, 2018; 27(Suppl 1): 25-38.

17. Du W, Wang LF, Shen Y, Zhang YZ, Ding WY, Ren LX. Long-term impacts of different posterior operations on curvature, neurological recovery and axial symptoms for multilevel cervical degenerative myelopathy. Eur Spine J, 2013; 22(7): 1594-1602.

18. Sielatycki JA, Armaghani S, Silverberg A, McGirt MJ, Devin CJ, O'Neill K. Is more lordosis associated with improved outcomes in cervical laminectomy and fusion when baseline alignment is lordotic? Spine J, 2016;16(8): 982-98

19. Lee CH, Lee J, Kang JD, et al. laminoplasty versus laminectomy and fusion for multilevel cervical myelopathy: a meta-analysis of clinical and radiological outcomes. J Neurosurg Spine, 2015; 22(6): 589-595.

20. Patel S, Glivar P, Asgarzadie F, Cheng DJW, Danisa O. The relationship between cervical lordosis and Nurick scores in patients undergoing circumferential vs. posterior alone cervical decompression, instrumentation and fusion for treatment of cervical spondylotic myelopathy. J Clin Neurosci, 2017; 45: 232-235.

21. Kimura A, Endo T, Inoue H, Seichi A, Takeshita K. Impact of axial neck pain on quality of life after laminoplasty. Spine (Phila Pa 1976), 2015; 40(24): E1292-E129

22. Wang M, Luo XJ, Deng QX, Li JH, Wang N. Prevalence of axial symptoms after posterior cervical decompression: a meta-analysis. Eur Spine J, 2016; 25(7): 2302-23

23. Healy AT, Lubelski D, West JL, Mageswaran P, Colbrunn R, Mroz TE. Biomechanics of open-door laminoplasty with and without preservation of posterior structures. J Neurosurg Spine, 2016; 24(5): 746-751.

24. Kihara S, Umebayashi T, Hoshimaru M. Technical improvements and results of open-door expansive laminoplasty with hydroxyapatite implants for cervical myelopathy. Neurosurgery, 2005; 57(4 Suppl): 348-3

25. Chen C, Yang C, Yang S, et al. Clinical and radiographic outcomes of modified unilateral open-door laminoplasty with posterior muscle-ligament complex preservation for cervical spondylotic myelopathy. Spine (Phila Pa 1976), 2019; 44(24): 1697-1704.

26. Sakaura H, Hosono N, Mukai Y, Iwasaki M, Yoshikawa H. Medium-term outcomes of C3-6 laminoplasty for cervical myelopathy: a prospective study with a minimum 5-year follow-up. Eur Spine J, 2011; 20(6): 928-933.

27. Kotani Y, Abumi K, Ito M, et al. Impact of deep extensor muscle-preserving approach on clinical outcome of laminoplasty for cervical spondylotic myelopathy: comparative cohort study. Eur Spine J, 2012; $21(8):$ 1536-1544.

28. Riew KD, Raich AL, Dettori JR, Heller JG. Neck pain following cervical laminoplasty: does preservation of the C2 muscle attachements and/or C7 matter? Evid Based Spine Care J, 2013; 4(1): 
42-53.

29. Lu J, Sun C, Bai JB, et al. Is correction of segmental kyphosis necessary in single-level anterior cervical fusion surgery? An observational study. Ther Clin Risk Manag, 2018; 15: 39-44.

30. Inui T, Inokuchi K, Watanabe Y, et al. Comparison of the operative time for open door laminoplasty using titanium plate spacers or hydroxyapatite block spacers: a retrospective study. J Orthop Surg Res,2019; 14(1): 475.

31. Li D, Hai Y, Meng X, Yang J, Yin P. Posterior open-door laminoplasty secured with titanium miniplates vs anchors: a comparative study of clinical efficacy and cervical sagittal balance. J Orthop Surg Res,2019; 14(1): 401.

\section{Figures}

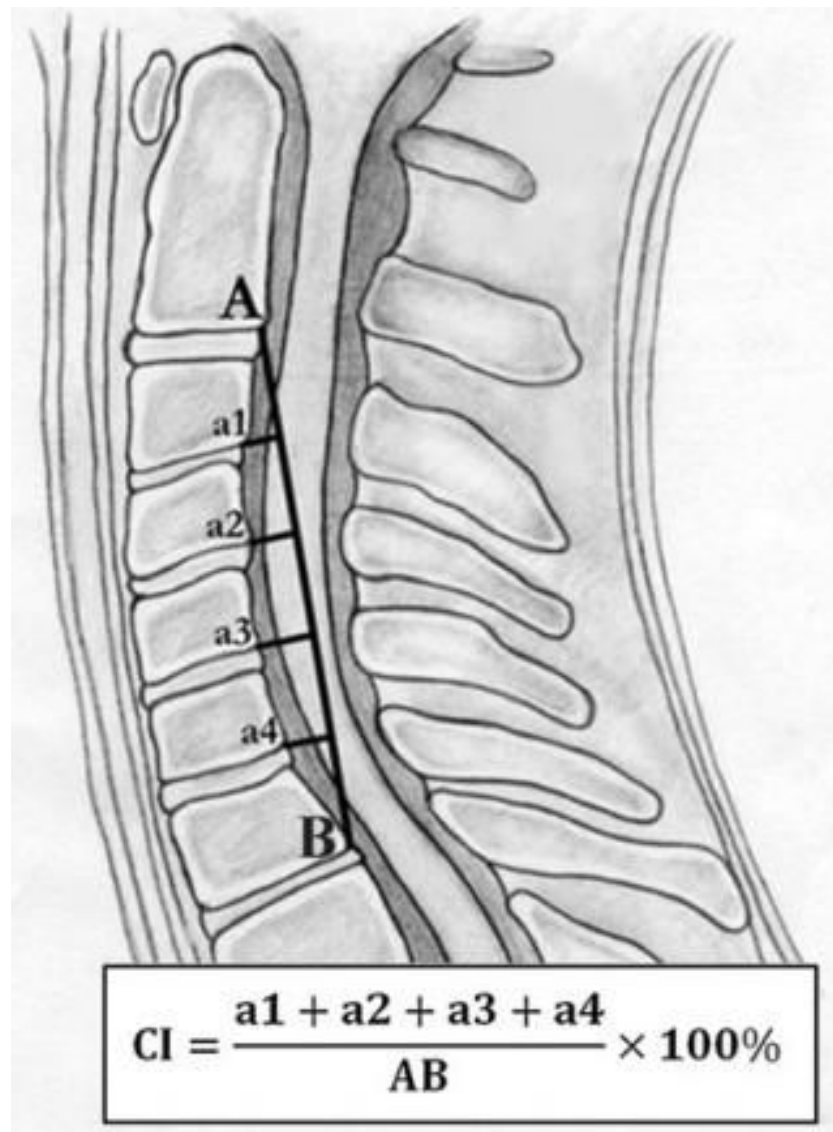

\section{Figure 1}

Calculation of the cervical curvature index $(\mathrm{Cl})$. 


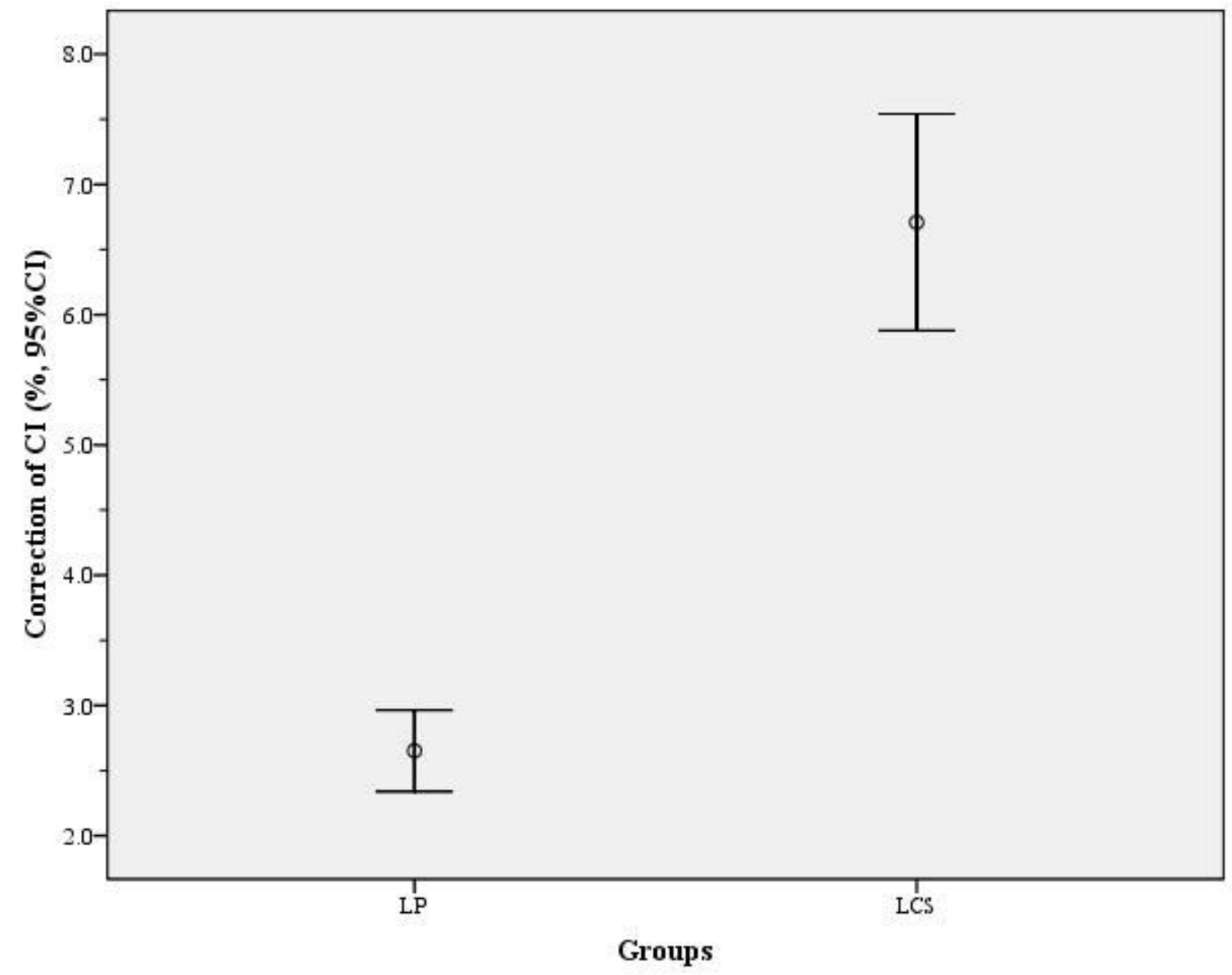

Figure 2

Loss of cervical curvature index in each group. The difference between the two groups for correction of $\mathrm{Cl}$ was statistically significant $(t=8.97, \mathrm{P}<0.001)$. 


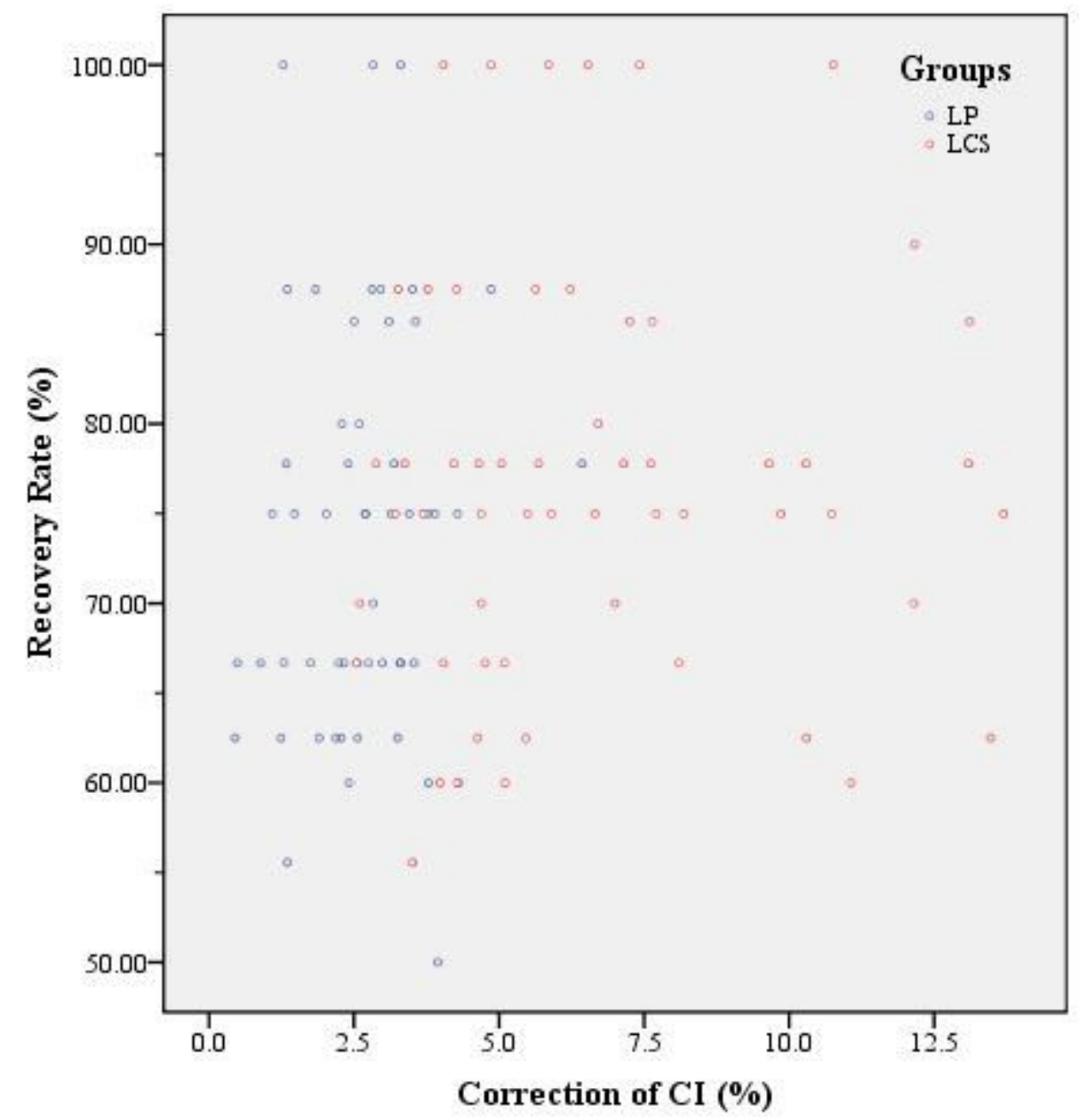

Figure 3

Correlation between correction of $\mathrm{Cl}$ and recovery rate $(r=0.14, \mathrm{P}=0.15)$. 


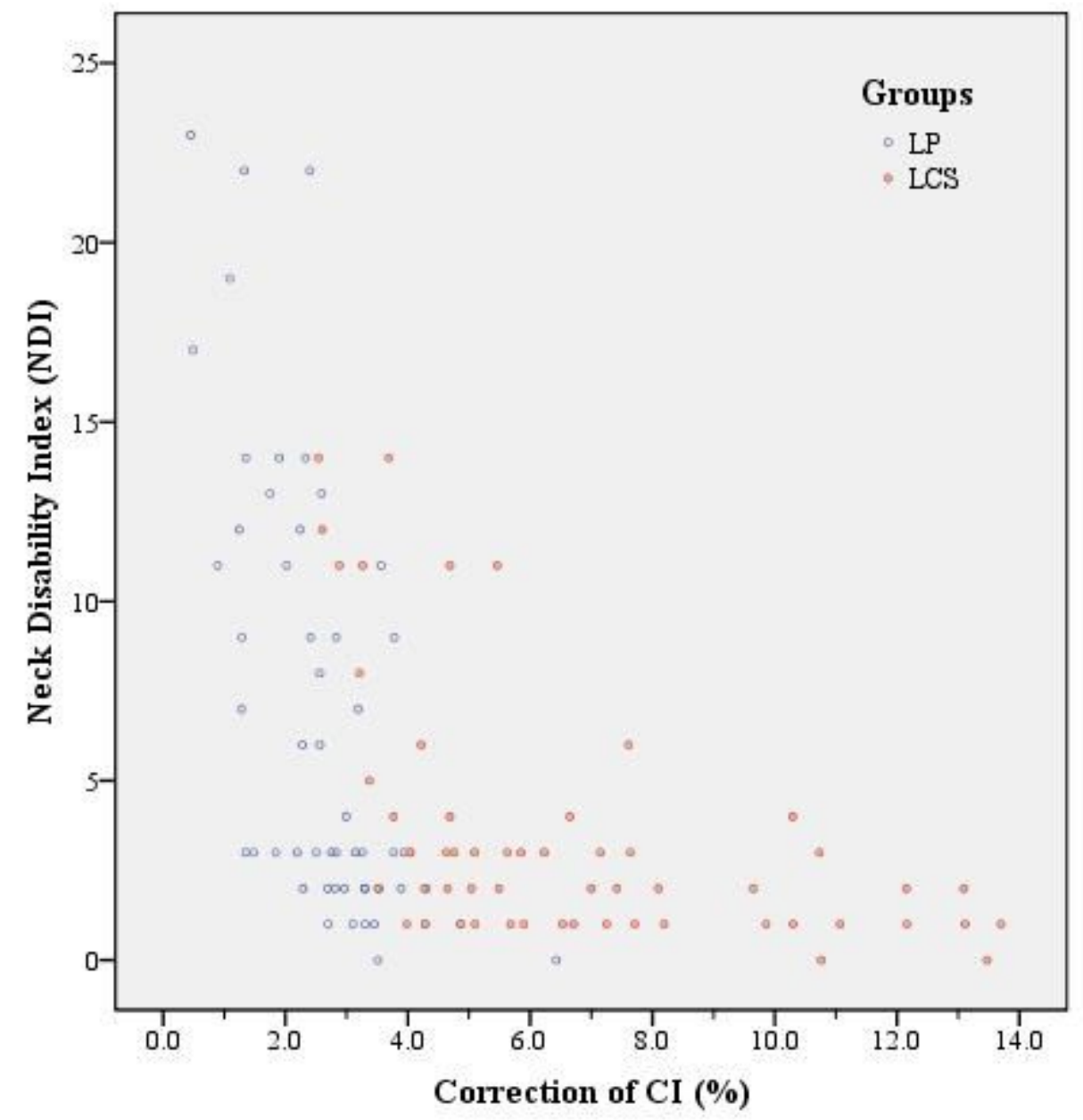

Figure 4

Correlation between correction of $\mathrm{Cl}$ and axial symptoms $(r=-0.51, \mathrm{P}<0.001)$. 